wir die sofortige Freilassung unserer Verwandten, tschechischer politischer Häft-

linge.

Prag - Brünn, den r. März 1976.

Unterschrieben:

Vilém Müller

Frantiska Müllerová

Marie Rusková

Ivan Rusek

Tatána Rusková

Rudolf Sabata

Ruzena Sabatová

Anna Sabatová

Jan Sabata

Václav Sabata

Eliska Skrenková (Hüblová)

Jana Tesarová

Brno, Jana Babáka 3/5

Brno, Jana Babáka 3/5

Brno, Drevarská 20

Brno, Drevarská 20

Brno, Drevarská 20

Dolenice c. 2

Dolenice c. 2

Praha 2, Anglická 8

Brno, nám. 28. rijna 20

Brno, Slovanská 5

Praha 5, Na Brezince I4

Praha 4, Pankrác II, is 50

\title{
Demonstrationsrecht und polizeiliche Überwachung
}

Der Staatsapparat hat seit jeher versucht, seine Kontroll- und Steuerungsmechanismen schon bei der politischen Meinungsbildung und Meinungsäußerung des Bürgers einzusetzen. Neuestes Beispiel dafür sind die in letzter Zeit zunehmenden polizeilichen Überwachungen politischer Demonstrationen selbst dann, wenn sie ordnungsgemäß angemeldet worden sind und "störungsfrei « verlaufen.

Dies geschah auch in dem folgenden von den Gerichten zu entscheidenden Fall: Mitte 1973 fand in Tübingen eine Demonstration von etwa 300 Personen gegen die geplante Änderung eines Hochschulgesetzes statt. Die Versammlung, die ordnungsgemäß angemeldet war und »störungsfrei« verlief, wurde von Kriminalbeamten überwacht und fotografiert. Eine Gruppe von Demonstranten, unter ihnen der Angeklagte, entdeckte einen fotografierenden Polizeibeamten und eilte zu ihm. Als der Polizeibeamte nach dem Eintreffen der Gruppe die Herausgabe des Films verweigerte, ergriff der Angeklagte den Fotokoffer und entfernte sich.

Das LG Tübingen' erblickte erst in dem späteren Behalten des Koffers ein strafbares Verhalten; die vorangegangene Wegnahme des Koffers sei dagegen durch Notwehr gerechtfertigt gewesen, weil der Polizeibeamte die Teilnehmer der Versammlung ohne gesetzliche Grundlage und unter Verletzung von Grundrechten (Art. 8 I GG) fotographiert hätte.

Dagegen ist nach dem $\mathrm{BGH}^{2}$ die Annahme von Notwehr rechtsfehlerhaft, weil das LG nicht berücksichtigt habe, daß die Polizeibeamten als Grund für das Fotografieren angaben, "man habe mit Hilfe der Lichtbilder die - unbekannten - Täter früherer Wandschmierereien und Vorlesungsstörungen, die als Teilnehmer an der Demonstration vermutet wurden, identifizieren wollen.« Unter diesen Umständen

\footnotetext{
Das Urteil des LG Tübıngen vom 18. 3. 1974 ist abgedruckt in: KJ 74, 4 I 8 ff. mit einer Anmerkung von Werner Robbers, $\mathrm{KJ} 74,425 \mathrm{ff}$.

$2 \mathrm{BGH}, \mathrm{NJW} 75,2075 \mathrm{f}$. und JZ 76, $31 \mathrm{f}$. mit einer Anmerkung von Walter Schmidt, JZ $76,32 \mathrm{f}$. Die Anmerkung yon W Robbers in der KJ wurde weder vom BGH noch von W Schmidt zur Kenntnis genommen.
} 
habe sich der Auftrag der Polizeibeamten, möglichst viele Teilnehmer der Demonstration zu fotografieren, im Rahmen der der Polizei nach $\$ 16_{3}$ StPO obliegenden Aufgabe gehalten, strafbare Handlungen zu erforschen.

Zentraler Punkt in beiden Urteilen ist die Frage nach der Rechtmäßigkeit der Úberwachung von politischen Demonstrationen durch fotografierende Polizeibeamte.

Das Fotografieren von Personen allein wegen ihrer Position als Versammlungsteilnehmer ist vom Gesetz nicht gedeckt und unzulässig, auch wenn es gemäß $\$ \mathbb{S}{ }_{16}$, I 52 II StPO, $\mathbb{} 24$ KUG um die Feststellung geht, ob sie als Täter vorangegangener Straftaten in Betracht kommen, deren thematischer Anlaß mit dem der Demonstration identisch ist.

Nur aufgrund eines konkreten Sachverhalts kann sich ein Verdacht ergeben, eine bestimmte Person könne eine strafbare Handlung begangen haben. Die objektive Tatbezogenheit einer Person resultiert also nicht aus den subjektiven Vorstellungen der Behörde, sondern die subjektive Vorstellung der Strafverfolgungsorgane hat sich an der objektiven Tatbezogenheit einer Person zu orientieren. Jemand ist nicht deshalb verdächtig, weil Polizeibeamte dies glauben, sondern weil seine Beziehung zur Tat objektiv auf seine Beteiligung hinweist ${ }^{3}$; erst aufgrund dieses objektiven Bezugs zur Tat kann sich eine subjektive Vorstellung des Verdachts i. S. der \$S ISz II, $163 \mathrm{StPO}$ gründen. Es handelt sich hier nicht um eine reine Ermessensentscheidung schon auf der Tatbestandsseite, die nur durch den Gesichtspunkı der Verhältnismäßigkeit, der ohnehin bei Grundrechtseingriffen grundsätzlich Anwendung zu finden hat, Einschränkungen erfährt, wie die Ausführungen von Schmidt ${ }^{4}$ in seiner Anmerkung vermuten lassen könnten.

Die bloße Demonstrationsteilnahme ist für sich gesehen ein Sozialakt, der hinsichtlich eventuell vorher begangener Straftaten nichts aussagt. Dies soll an einem unpolitischen Beispiel verdeutlicht werden', das zeigt, daß der Verdacht der Strafverfolgungsorgane nur auf zusätzliche objektive Merkmale in der Person des Teilnehmers gestützt werden kann: Bei einem Kraftfahrer, der eine Gaststätte verläßt, um seine Fahrt fortzusetzen, ist dies ein noch zweifelhaftes Indiz und noch kein "zureichender tatsächlicher Anhaltspunkt« i. S. der $\$ \$ 15_{2}$ II, i63 StPO für Alkoholgenuß, weil man an derartigen Stätten keineswegs nur Alkohol zu sich nimmt. Freilich kann es sein, daß der die Gaststätte verlassende Fahrer tatsächlich angetrunken ist; dies trifft aber nicht mit Notwendigkeit auf jeden zu, der eine Gaststätte verläßt, und ist deshalb im rechtlichen Sinne für einen Verdacht irrelevant. Ebensowenig wie das Verlassen einer Gaststä̈tte als Anknüpfungspunkt für den Verdacht der Trunkenheit ausreicht, vielmehr noch andere objektivierbare Momente in der Person hinzukommen müssen (etwa schwankende Gehweise), so reicht auch die Teilnahme an einer Demonstration, die nur thematischen Bezug zu vorher begangener Straftaten hat, für den Verdacht der Begehung dieser Taten nicht aus. Ein Verdacht, der nur an die Demonstrationsteilnahme ais solche anknüpft, ist eine unzulässige Diskriminierung, die lediglich auf ein Pauschalurteil gegründet ist. Es bedarf vielmehr weiterer, konkreter Anhaltspunkte, die die Tatbeteiligung vermuten lassen.

Stattdessen verwendet der BGH einen Kunstgriff, der darin besteht, daß anstelle einer Begründung des Verdachts eine Verdachtsbehauptung gesetzt und diese in Form einer Begründung vorgetragen wird: Jemand ist einer Straftat verdächtig, weil

\footnotetext{
3 Vgl. F. Geerds, Ưber strafprozessuale Maßnahmen, Insbesondere Entnahme von Blutproben be1 Verdacht der Trunkenhert am Steuer, in: GA 65, 32r (327f.). 
er an einer Versammlung teilnimmt, an der wegen ihres thematischen Bezugs eventuell auch Täter früherer Straftaten teilnehmen könnten. Weil aber eventuell auch Straftäter teilnehmen könnten, sind dadurch nicht alle Demonstranten verdächtig. Jemand kann nur verdächtig sein infolge eines Umstandes, der in ihm liegt, aber nicht infolge eines Umstandes, der von außen hinzukommt, und auf den er keinen Einfluß hat. Warum in jedem Teilnehmer der Versammlung ein Verdächtiger zu vermuten ist, wird nicht begründet. Damit hat der BGH als Ergebnis gerade das vorweggenommen, was erst für jeden einzelnen Teilnehmer gesondert zu begründen gewesen wäre. Denn sonst wären die Teilnehmer jeder Demonstration verdächtig, weil sich stets eine mögliche Verbindung zu Straftaten konstruieren läßt. Mit Hilfe der Konstruktionsbrücke des $\mathbb{\$}{ }_{16} 6_{3}$ StPO könnten Demonstrationsteilnehmer stets mit der Begründung fotografiert werden, dies geschehe im Rahmen von Ermittlungsverfahren wegen Straftaten ${ }^{6}$, die sich im Zusammenhang mit Demonstrationen ereignet haben könnten. Eine Vermutung, daß die Straftäter sich auch an der Versammlung beteiligen werden, kann sich aber bei Fehlen weiterer Umstände noch nicht zu einem rechtlich faßbaren und relevanten Verdacht i.S. des $\mathbb{1}$ 163 StPO verdichten. Folgte man konsequent dem BGH, so könnte die Polizei jede Person in jeder Menschenansammlung mit der Begründung fotografieren, es handele sich um eine Strafverfolgungsmaßnahme, weil nicht ausgeschlossen werden könne, daß sich in einer Menschenmenge auch Straftäter aufhielten. Es gibt aber keine Rechtsvermutung für einen Verdacht bei einem grundsätzlich zulässigen Tun. Die Teilnahme an einer Demonstration allein darf demnach nicht als Grund für den Verdacht der Beteiligung an einer strafbaren Handlung gewertet werden.

Der BGH stützt die Zulässigkeit von Fotoaufnahmen auch auf $\$ 23 \mathrm{I} \mathrm{Nr} .3 \mathrm{KUG}$, wonach grundsätzlich Bilder von Versammlungen verbreitet und aufgenommen werden dürfen. Hier wirkt sich bei der Subsumtion der methodische Fehler des BGH aus, das allgemeine Persönlichkeitsrecht nicht im Zusammenhang mit der jeweiligen gesellschaftlichen Situation zu sehen, auf die es Anwendung finden soll ${ }^{7}$. So hätte der BGH die Frage erörtern müssen, ob durch $\$ 23$ I Nr. 3 KUG nur solche Aufnahmen zulässig sind, bei denen der Fotografierende die Demonstration als Ganzes aufnehmen will, innerhalb dessen die abgebildeten Personen nur notwendiges Beiwerk, d. h. Nebenfiguren, bilden ${ }^{8}$, nicht aber gezielt Aufnahmen jedes einzelnen Demonstranten.

Die Demonstrationsfreiheit ist gerichtet auf den gesellschaftlichen Meinungsbildungsprozeß und hat die Funktion, die bereits in Art. s GG strukturell angelegte Offnung zum Kollektiven politisch folgenreich zu erweitern. ${ }^{9}$ Adressat der Demonstration ist somit die Offentlichkeit. Der Staatsapparat darf daher die geäußerten Meinungen nur in der Weise zur Kenntnis nehmen, wie sie von der Intention der Demonstrationsteilnehmer gedeckt ist, nämlich als Diskussionsbeitrag zum demokratischen Meinungsbildungsprozeß.

\footnotetext{
6 Solche herauszusuchen, dürfte in der heutıgen politıschen Situatıon keıne Schwierigkeıt bereıten, wo z. B. das Beschriften von Hauswänden oder Ankleben von Plakaten an Brückenpfeiler von verschıdenen Oberlandesgerıchten als Sachbeschädigung gewertet werden; OLG Karlsruhe, JZ 75, 642; OLG Hamburg, NJW 75, 198I. Möglicherwesse werden schon heute Demonstranten gegen $\$ 2$ I 8 StGB mit der Begründung fotografiert, es könne nıcht ausgeschlossen werden, daß sıe sich bereıts als Täter oder Tatbeteiligte gem. $\$ 218$ StGB strafbar gemacht hätten.

7 Uber die Möglichkeıt von Fotoaufnahmen von Demonstranten vgl. auch H. U. Evers, Das allgemene Persönlichkeıtsrecht ım öffentlichen Recht und die Aufnahme von Lıchtbildern zur Uberwachung von Versammlungen, ın: Festschrift für R. Reınhardt, S. 377 f., dem, ohne den wichtıgen gesellschaftlichen Bezug des Persönlichkeitsrechts zu erkennen, es deshalb auch nicht gelingt, die Funktion der Versammlungsfreiheıt für den demokratıschen Willensbildungsprozeß zu diskutıeren.

8 Vgl. den Hinwers be1 Ingo von Münch, In: JuS 66, 404 (408 Fn. 20).

9 Vgl. dazu Ulrich Scbwäble, Das Grundreche der Versammlungsfreihe1t (Art. 8 GG), Schriften zum Offentlichen Rechs Bd. 275, Berlin 1975, S. 98
} 
An diesem Zweck ist auch der Umfang des Persönlichkeitsrechts der Demonstranten zu bestimmen. Der einzelne Versammlungsteilnehmer will gerade so viel an Einbuße seines Persönlichkeitsrechts hinnehmen, wie für den politischen Prozeß notwendig ist. Im Rahmen des $\$ 23$ I Nr. 3 KUG können somit diejenigen Zwecke keine Geltung beanspruchen, die nicht in den Bezugsrahmen öffentlicher demokratischer Diskussion eingeordnet werden können. Demnach läßt sich $\ 23 \mathrm{I} \mathrm{Nr} .3$ KUG nicht als Rechtsgrundlage für die Anfertigung der Aufnahmen von Einzelpersonen zur behördeninternen Verwendung heranziehen.

Infolge der Rechtsprechung des BGH riskiert jeder Demonstrationsteilnehmer, daß er fotografiert wird. Das Ergebnis ist, daß abweichende politische Meinungsäußerungen in der Offentlichkeit nicht mehr möglich sind, ohne daß die Gefahr einer nachteiligen Verwendung besteht. Meinungen können nur noch in privaten Zirkeln und Grüppchen frei ausgetragen werden. Die Teilnehmerschaft an Demonstrationen reduziert sich auf den Kernbestand der um jeden Preis Utberzeugten, die bereit sind, für ihre Úberzeugungen alle Nachteile (z. B. auch die Nichtübernahme in den öffentlichen Dienst) auf sich zu nehmen, was dem Staatsapparat wiederum ermöglicht, Demonstrationen die Legitimität abzusprechen (Stichwort: »Berufsdemonstranten «). Der andere Teil, der, aus welchen Gründen immer, nicht dazu bereit ist, wird entweder in politischen Konformismus absinken oder im Untergrund anonyme Agitation betreiben. Die Kausalkette ist perfekt: Das Mittel zur Aufklärung von Straftaten (Fotoaufnahmen von Demonstranten) setzt neue Kausalreihen zur Begehung neuer Straftaten und rechtfertigt somit weitere Úberwachungsmaßnahmen des Staatsapparates. Tatsächlich können auf diese Weise abweichende politische Ansichten und Motive kriminalisiert und damit politische Freiheitsrechte eingeengt werden.

Zunehmend vollzieht sich die Filtrierung und Kontrolle des Willensbildungsprozesses nicht mehr allein durch gewaltsame Eingriffe in das Demonstrationsrecht, sondern durch Mechanismen, die disziplinierend auf den Prozeß der Transformation des Willensentschlusses in faktische Handlung wirken. Bestand im absolutistischen Staat der Eingriff des Staatsapparates vor allem in physischer Gewaltanwendung, so daß die Grundrechtsidee des Liberalismus nur die Handlungsausübung als Schutzbereich der Freiheitsrechte sehen konnte, so ist heute die Steuerungsfähigkeit des Staatsapparates subtiler geworden und kann soweit gehen, daß bestimmte kollektive Interessen, die für den Produktionsprozeß irrelevant sind, ihre Artikulationsfähigkeit überhaupt verlieren ${ }^{10}$. Die Ausschaltung abweichender Motive und Ansichten aus dem politischen Willensbildungsprozeß durch den Staatsapparat beruht nicht mehr allein auf dem groben und schwer zu legitimierenden, weil die "Friedensordnung " gefährdenden, Instrumentarium der Beschränkung der tatsächlichen Handlungsausübung durch Verbote und direkte Einschränkungen subjektiver Rechte, sondern die Kontrollfunktionen werden schon früh bei der Willensbildung und Entschlußfreiheit eingesetzt.

So kann in die Versammlungsfreiheit heute nicht nur durch äußere unmittelbare, physische Einwirkungen des Staatsapparates auf Durchführung und Ablauf der Demonstration (etwa durch Verbot und Auflösung) eingegriffen werden, sondern in

10 Dazu die Analysen von Claus Offe, Strukturprobleme des kapıtalistıschen Staates, Aufsätze zur Politischen Sozıologıe, 2. Aufl. Frankfurt I973. Uber die disziplinierenden Mechanismen und Steuerungsmöglichkeıten, die in den Instıtutıonen der politıschen Bedürfnısartikulatıon und dem System der politischen Willensbildung selbst eingebaut sind, wie etwa in den Parteren, Gewerkschaften usw., gibt die Analyse von Offe über: "Politısche Herrschaft und Klassenstrukturen. Zur Analyse spätkapıtalistıscher Gesellschaftssysteme " Aufschluß; dort insbesondere das Kapitel: „Herrschaftsmechanismen im System der polituschen Willensbildung«, in: Kritusche Studien zur Politikwıssenschaft. Eine Einführung in ihre Probleme, S. I55-189 (insbes. S. $164 \mathrm{ff}$.), 3. Aufl. Frankfurt 1971 . 
gleicher Weise durch mittelbare Einwirkungen, so daß erst gar nicht der Entschluß entsteht, an einer Versammlung teilzunehmen oder weiter teilzunehmen. Diese mittelbare Eingriffsart ist besonders gefährlich, weil nicht direkt die Grundrechtsausübung angetastet wird, die politischen Freiheitsrechte also formal gewährleistet bleiben, sondern der Eingriff eine Stufe vor der Handlungsvollziehung, nämlich auf der Stufe der vorangehenden Entschlußfassung einsetzt. Der Eingriff braucht nicht in unmittelbarer Gewalt nach außen in Erscheinung zu treten, sondern kann in der inneren Sphäre der Willensbildung ansetzen, wodurch es ermöglicht wird, Grundrechtseingriffe versteckt ohne unmittelbare Störung der gesellschaftlichen »Friedensordnung « vorzunehmen.

Die Entschlußfreiheit zur Teilnahme an einer Versammlung ist nicht mehr frei, wenn der einzelne Teilnehmer oder Teilnahmewillige weiß oder damit rechnen muß oder während der Teilnahme bemerkt, daß seine Anwesenheit und Äußerungen von Staats wegen nicht als Entgegennahme eines Diskussionsbeitrages im Rahmen des demokratischen Meinungsbildungsprozesses gewertet werden, sondern daß Kriminalbeamte ihn zum Zwecke eventueller Strafverfolgung oder gar zur Anlage von Demonstrantenkarteien fotografieren. Letzteren Zweck zu beweisen, muß immer scheitern, weil die betroffenen Demonstranten insoweit die Beweislast trifft und anzunehmen ist, daß den in Betracht kommenden Beamten keine Aussagegenehmigung hinsichtlich des Verwendungszwecks der Fotoaufnahmen erteilt wird "1 , so daß ein Grundrechtseingriff in diesem Fall ohne Rechtsschutz des Betroffenen bleibt.

Die Erkenntnis, daß von Seiten des Staatsapparates die Kontrollfunktion und Ausschaltung mißliebiger Willensbildung heute schon früh bei der Entschlußfreiheit einsetzt, und daß die innere Entschlußfreiheit tatsächlich manipulierbar und damit Restriktionen zugänglich ist, muß zu einer Erweiterung des Schutzkreises der politischen Freiheitsrechte führen. Die Reichweite des Grundrechtsschutzes muß funktional der Eingriffsart sein, d. h. ändert sich die Art der Eingriffe, muß sich mit ihr der Schutzbereich des Grundrechts erweiteren, damit nicht die materielle Substanz des Grundrechts ausgehöhlt wird. Die jeweiligen Grundrechtsgewährleistungen erfassen demnach in gleichem Umfang neben der äußeren Handlungsausübung ebenso die innere Willens- und Entschlußfreiheit. Die Folge ist, daß auf die Entschlußfreiheit nur insoweit Einfluß genommen und sie nur in dem Maße eingeschränkt werden darf, wie die Freiheitsausübung selbst ${ }^{12}$. Entschließt sich nun ein Teilnahmewilliger wegen der möglichen Bildaufnahme, an der Demonstration nicht oder nicht mehr weiter teilzunehmen, so hat sich die Einflußnahme auf die Entschlußfreiheit mittelbar auf die Versammlungsteilnahme und damit auf die Freiheitsausübung ausgewirkt. Dies bedeutet einen unzulässigen Eingriff in den äußeren Freiheitsgebrauch, der dem Schutz des Art. 8 GG unterliegt.

Die Aberkennung des Notwehrrechtes des Demonstrationsteilnehmers gegenüber fotografierenden Polizeibeamten durch den BGH reiht sich lückenlos in zahlreiche Versuche des Staatsapparates und deren Absicherung durch die Rechtsprechung ein, eine gesellschaftliche Situation zu schaffen, die sich dadurch kennzeichnet, politische Auseinandersetzungen in ein Prokrustesbett zu zwängen und politische Freiheitsrechte einzuengen. Es geht aber nicht nur um Rechtsstaatlichkeit und Entlarvung von "Verfassungsfeinden « (wie die Entscheidungen zu den Berufsverboten suggerieren wollen), sondern auch und vor allem um die Offenheit der geistigen Auseinandersetzung und des politischen Willensbildungsprozesses.

Hanns-Peter Hartlieb

II Vgl. auch die Urteilsbegründung des LG Tübıngen, in: KJ 74, 4 I8 (424)

12 Ahnlich auch schon K. Kübler, Nochmals: Die fotografierenden Verfassungsschützer, in: JuS 66, 319 (320). 